\title{
Molecular Detection of Rickettsia felis and Rickettsia bellii in Mosquitoes
}

\author{
Jilei Zhang, ${ }^{1,2}$ Guangwu Lu, Jing Li, ${ }^{1}$ Patrick Kelly, ${ }^{3}$ Min Li, ${ }^{1}$ Jiawei Wang, ${ }^{1}$ \\ Ke Huang, ${ }_{1}$ Haixiang Qiu, Jinfeng You, ${ }_{1}^{1}$ Rong Zhang, ${ }^{1}$ Yaoyao Wang, ${ }^{1}$ Yuanyuan Zhang, \\ Hongzhuan Wu, ${ }^{4}$ and Chengming Wang ${ }^{1,5}$
}

\begin{abstract}
To add to the limited information on Rickettsia in mosquitoes in China, we carried out a PCR survey on convenience samples of 3051 mosquitoes collected with hand nets in and around domestic dwellings in 25 provinces. Five species of mosquitoes were identified: Culex pipiens pallens $(n=1620)$, Aedes albopictus (806), Armigeres subalbatus (377), Anopheles sinensis (168), and Culex tritaeniorhynchus (80). A Rickettsia nestedPCR targeting the variable domain of gltA showed Rickettsia felis in four mosquito species of 16 provinces $C x$. pipiens pallens (1.8\%, 29/1620); Ae. albopictus (1.2\%, 10/806); An. sinensis (1.2\%, 2/168); and Ar. subalbatus $(2.1 \%, 8 / 377)$. Rickettsia bellii was also widespread, occurring in 12 provinces and 2 species: Cx. pipiens pallens $(4.3 \%, 69 / 1620)$ and An. sinensis $(0.6 \%, 1 / 168)$. R. felis and $R$. bellii were found in almost similar numbers in female [1.5\% (27/1809) and $1.2 \%(21 / 1809)$, respectively] as in male mosquitoes [1.8\% (22/1242) and $4.0 \%$ (49/1242), respectively]. Our results indicated that mosquitoes in China are widely infected with $R$. felis, the agent of human flea-borne spotted fever, and that $R$. bellii can also occur outside of the Americas and its usual tick hosts.
\end{abstract}

Keywords: Rickettsia felis, Rickettsia belli, PCR, mosquito, China

\section{Introduction}

$\mathbf{T}$ HE GENUS RICKETTSIA comprises gram-negative obligate intracellular $\alpha$-proteobacteria that have been divided into four clades or groups based on phylogenetic analysis (Gillespie et al. 2007, 2008). The spotted fever group and typhus groups are important human pathogens worldwide that are principally transmitted by ixodid ticks, and fleas and lice, respectively. The major clinical signs of infection include fever, rash, headache, chills, myalgia, and malaise (Blanton and Walker 2017, Carvalho et al. 2017). Some Rickettsia, for example, Rickettsia africae, cause mild or subclinical disease while other species, such as Rickettsia prowazekii and Rickettsia rickettsii, can cause severe disease and high mortality (Mediannikov et al. 2013, Blanton and Walker 2017). The Rickettsia have been divided into four clades or groups based on phylogenetic analysis (Gillespie et al. 2007, 2008). The transitional group, represented by Rickettsia felis, and the ancestral group, represented by Rickettsia bellii, are most widely reported to be transmitted by fleas and a wide range of tick species in the Americas, respectively (Krawczak et al. 2018).

Since 1982, at least 10 recognized and uncharacterized species of spotted fever group rickettsiae have been identified in China (Fang et al. 2015). One of the more recently described, $R$. felis, is the agent of flea-borne spotted fever in people. Although originally associated with the cat flea (Ctenocephalides felis) (Blanton and Walker 2017), it has now been found to occur in a variety of mosquitoes: Aedes spp., Anopheles spp., and Mansonia uniformis in Africa (Socolovschi et al. 2012a, 2012b, Mediannikov et al. 2013) and Anopheles sinensis and Culex pipiens in China (Zhang et al. 2014). Furthermore, it has been shown that Anopheles gambiae can be infected with $R$. felis and can transmit the

${ }^{1}$ College of Veterinary Medicine, Yangzhou University, Yangzhou, China

${ }^{2}$ Division of Gastroenterology and Hepatology, Department of Medicine, University of Illinois at Chicago, Chicago, Illinois.

${ }^{3}$ Ross University School of Veterinary Medicine, Basseterre, West Indies.

${ }^{4}$ Program in Microbiology, Alabama State University, Montgomery, Alabama

${ }^{5}$ College of Veterinary Medicine, Auburn University, Auburn, Alabama. 
organism during feeding, indicating it to be a potential vector (Dieme et al. 2015). Finally, novel Rickettsia spp. have been found in An. gambiae and Anopheles melas in Africa (Socolovschi et al. 2012a), Cx. pipiens (Zhang et al. 2014) and An. sinensis and Culex tritaeniorhynchus (Guo et al. 2016) in China, and M. uniformis, C. pipiens, and Aedes esoensis in Korea (Maina et al. 2017).

In previous studies, we have reported Rickettsia sp. in mosquitoes from Yangzhou, China (Zhang et al. 2014, 2016). To add to the available data on Rickettsia in different mosquito species in China, we collected samples of mosquitoes from 25 provinces and tested them for Rickettsia spp. by PCR.

\section{Materials and Methods}

\section{Sample collection}

Between July and September 2014, student volunteers from Yangzhou University used hand nets to collect convenience samples of mosquitoes in their primary homes located in 26 cities in 25 provinces or municipalities in China. Mosquitoes were immediately placed individually in sterile tubes containing $400 \mu \mathrm{L}$ DNA/RNA stabilization reagent for blood/bone marrow (Roche Molecular Biochemicals, Indianapolis, IN). Then, samples were transported to Yangzhou University College of Veterinary Medicine at room temperature, where they were stored at $-80^{\circ} \mathrm{C}$ until DNA extraction.

\section{DNA extraction}

Each mosquito was thawed at room temperature and homogenized individually in a shaker (Bertin Technologies, France) with four $3.0 \mathrm{~mm}$ ceramic beads for two short durations of $15 \mathrm{~s}$ (3160 $\mathrm{g}$ with a 15-s break in between) each. The High-Pure PCR Template Preparation Kit (Roche Molecular Biochemicals) was used to extract total nucleic acids in $100 \mu \mathrm{L}$ elution buffer according to the manufacturer's instructions and as described before (Wei et al. 2014, Zhang et al. 2014).

\section{Mosquito identification}

The species and gender of the mosquitoes were determined using standard morphological criteria (Harbach 2013). Species identification was further confirmed with PCR and gene sequencing by using the primers (LCO1490: 5'-GGTCAA CAAATCATAAAGATATTGG-3', and HCO2198: 5'-TAAA CTTCAGGGTGACCAAAAAATCA-3') targeting the mitochondrial cytochrome c oxidase subunit I gene $(C O I)$ as described earlier (Folmer et al. 1994, Wang et al. 2012).

\section{Identification of Rickettsia spp. in mosquitoes}

A previously validated gltA-based FRET-qPCR (Zhang et al. 2014) was used to detect Rickettsia spp. in the individual mosquitoes. Samples positive for Rickettsia spp. based on the FRET-qPCR and high-resolution melting curve analysis were further amplified with primers of a nested-PCR targeting the gltA gene as described previously (Zhang et al. 2014) and subsequent sequencing of amplicons (BGI, Shanghai, China). Identification of Rickettsia spp. using nested-PCR targeting the gltA has been shown to be as reliable as identification based on MLST (gltA, ompA, ompB, and sca4 genes) (Zhang et al. 2014, 2016). The PCR amplicon of
R. felis identified previously (Zhang et al. 2014) served as the positive control, while sterile water was used as the negative control.

\section{Phylogenetic analysis}

The retrieved Rickettsia sequences of the present study and the reference sequences from GenBank were aligned using the MEGA 6.0 software (Fig. 2). Based on these alignments, phylogenetic trees were constructed by the neighbor-joining method using the Kimura 2-parameter model with MEGA 6.0. Bootstrap values were calculated using 500 replicates (Zhang et al. 2018).

\section{Statistical analysis}

Chi-squared test was used to compare the prevalence of Rickettsia spp. between different mosquito species and in different provinces of China. A value of $p<0.05$ was considered to be significant.

\section{Results}

\section{Mosquito species identified}

Of the 3051 mosquitoes captured (Fig. 1), 59.3\% (1809/ 3051) were female and $40.7 \%$ (1242/3051) were male (Table 1). Five mosquito species were identified using morphological criteria and PCR followed by DNA sequencing: Aedes albopictus $(n=806,26.4 \%)$, An. sinensis $(n=168$, $5.5 \%)$, Armigeres subalbatus $(n=377,12.4 \%)$, Culex pipiens pallens $(n=1620,53.1 \%)$, and Culex tritaeniorhynchus $(n=80,2.6 \%)$. The Culex spp. $(n=1700)$ were found in all 26 cities studied, except for Zhuzhou in Hunan province (Table 1 and Fig. 1). Culex tritaeniorhynchus was only identified in Guizhou province in the southwest of China (Fig. 1).

\section{Rickettsia spp. in mosquitoes}

Overall, 3.9\% (119/3051) of the mosquitoes were found positive in the Rickettsia FRET-qPCR. Positive mosquitoes were found in all but four provinces (21/25), including Hebei, Liaoning, Jiangxi, and Fujian. The prevalences of Rickettsia spp. in mosquitoes from Heilongjiang (24.6\%, 27/110), Shanghai (15.8\%, 19/120), and Beijing (16.8\%, 20/119) were significantly higher than the other provinces $(0 \%$ of $0 / 451$ to $5.2 \%$ of $6 / 116 ; p<0.01)$. Significantly higher numbers of $C x$. pipiens pallens $(6.1 \%, 98 / 1620)$ were found positive when compared with the other species: Ae. albopictus $(1.2 \%, 10 /$ 806), An. sinensis (1.8\%, 3/168), Ar. subalbatus $(2.1 \%, 8 /$ $377)$, and $C x$. tritaeniorhynchus $(0 \%, 0 / 80)(p<0.05)$. Also, significantly more male $(5.7 \%, 71 / 1242)$ than female $(2.7 \%$, 48/1809) mosquitoes were positive for Rickettsia spp. (Table 1) $(p<0.01)$.

Sequencing of the amplicons of the PCR targeting the variable domain of the gltA indicated that there were two Rickettsia spp. in the mosquitoes: R. felis and R. bellii. The gltA sequences of all the $R$. felis species were identical to one another (given the GenBank accession number MK061744) and a reference $R$. felis strain from Brazil (KX446943). There was only one nucleotide mismatch with the $R$. felis previously identified from China (KJ440519) (Fig. 2). Similarly, all the $R$. bellii sequences were identical to one another (given the 


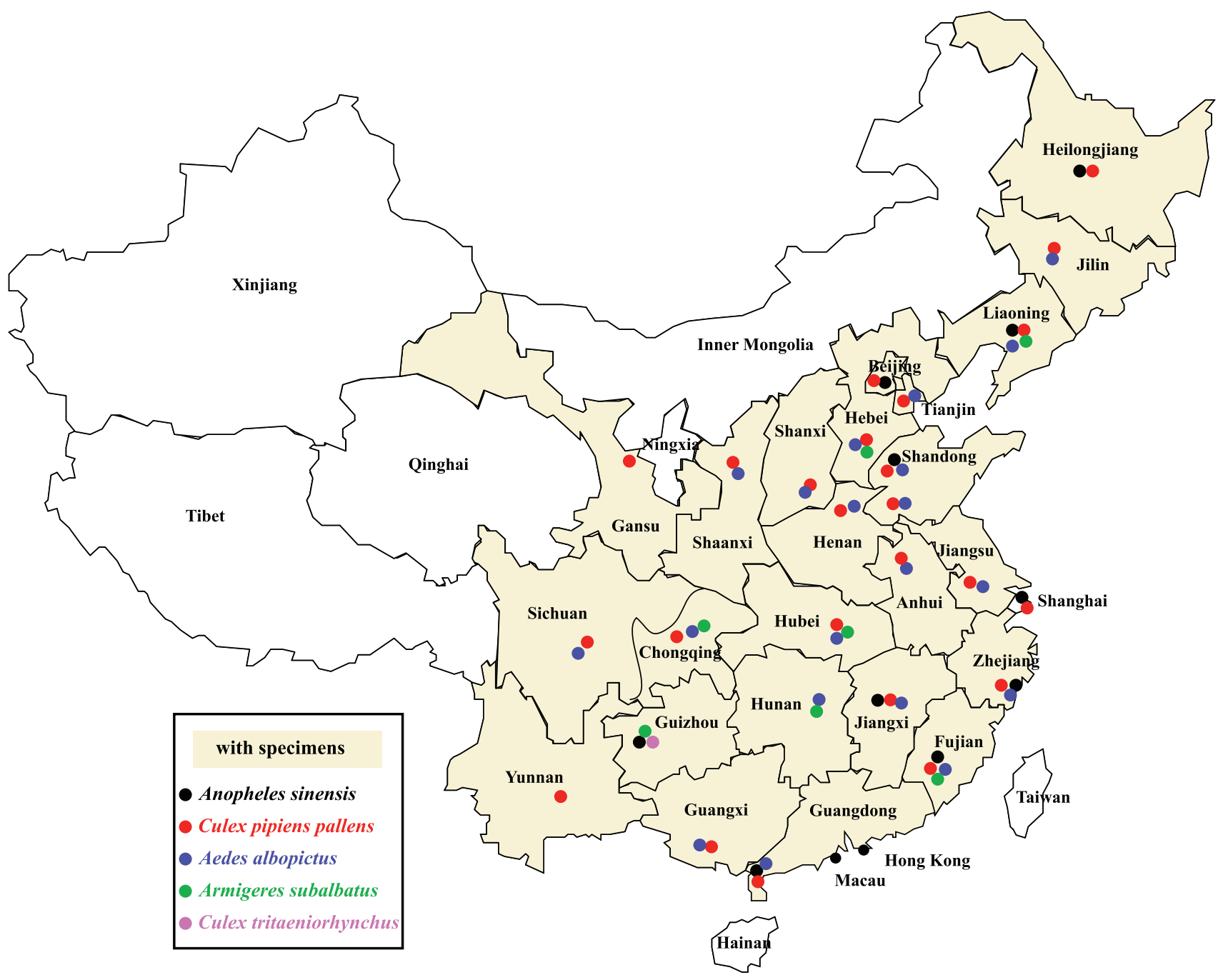

FIG. 1. Mosquitoes collected in and around domestic dwellings in China. The mosquito samples $(n=3051)$ were collected from 25 provinces/municipalities in northern, southern, and eastern China. Five species of mosquito were collected, mainly Anopheles sinensis, Culex pipiens pallens, Aedes albopictus, Armigeres subalbatus, and Culex tritaeniorhynchus. Color images are available online.

GenBank accession number MK697586) and to reference strains in GenBank from China and South Korea (KU586331 and KY799071).

The $R$. bellii species were found more commonly $(2.3 \%, 70 /$ 3051) than $R$. felis $(1.6 \%, 49 / 3051)$. In fact, $R$. felis was found in four mosquito species (Cx. pipiens pallens, 1.8\%, 29/1620; Ae. albopictus, 1.2\%, 10/806; An. sinensis, 1.2\%, 2/168; and Ar. subalbatus, $2.1 \%, 8 / 377)$, while $R$. bellii was only detected in Cx. pipiens pallens $(4.3 \%, 69 / 1620)$ and An. sinensis $(0.6 \%$, 1/168). $R$. felis appeared more widespread, being present in 16 provinces, while $R$. bellii was identified in only 12 provinces. Similar numbers of female (R. felis: $1.5 \%, 27 / 1809 ;$ R. bellii: $1.2 \%, 21 / 1809$ ) and male mosquitoes (R. felis: 1.8\%, 22/1242; R. bellii: $4.0 \%, 49 / 1242$ ) were positive for each organism. Any concurrent infections were not detected.

\section{Discussion}

There are around 41 genera and 3500 species and subspecies of mosquitoes worldwide with at least 390 species occurring in China and a few new species still not being identified (Wang et al. 2012, Guo et al. 2018). All five mosquito species collected in our study feed on humans. Some of the mosquitoes also feed on other animals, such as Cx. pipiens pallens on birds, An. Sinensis on cattle and buffalo, and Cx. Tritaeniorhynchus on pigs and cattle (Wang et al. 2012). Their capture in our study, then, might have been as a result of livestock being kept in the vicinity of the students' houses. The species most commonly collected, $C x$. pipiens pallens followed by Ae. albopictus, were also found in most of the provinces studied, which confirms previous reports on these species' high prevalence and widespread distribution in China (Cao et al. 2011, Wang et al. 2011, Feng et al. 2012, Guo et al. 2014, Huang et al. 2015).

Previously, we described $R$. felis in mosquitoes from Jiangsu Province in China (Zhang et al. 2014). In our study, we show that the organism is very widespread in China, occurring in 16 of 25 provinces. To date, $R$ felis has been recovered from An. sinensis and Cx pipiens pallens in China (Zhang et al. 2014) and An. gambiae (Socolovschi et al. 
Table 1. Positivity of Rickettsia in Mosquitoes Collected in This Study

\begin{tabular}{|c|c|c|c|c|c|c|c|}
\hline Province & City & Species & Gender & No. & Total & Rickettsia felis & Rickettsia bellii \\
\hline Zhejiang & Wenzhou & $\begin{array}{l}\text { An. sinensis } \\
\text { Cx. p. pallens } \\
\text { Ae. albopictus }\end{array}$ & $\begin{array}{l}\mathrm{F} \\
\mathrm{M} \\
\mathrm{F} \\
\mathrm{M} \\
\mathrm{F} \\
\mathrm{M}\end{array}$ & $\begin{array}{r}24 \\
70 \\
3 \\
11 \\
8 \\
4\end{array}$ & 1 & & 1 \\
\hline Shanghai & Shanghai & $\begin{array}{l}\text { Cx. p. pallens } \\
\text { An. sinensis }\end{array}$ & $\begin{array}{l}\mathrm{F} \\
\mathrm{M} \\
\mathrm{F}\end{array}$ & $\begin{array}{r}80 \\
37 \\
3\end{array}$ & $\begin{array}{r}11 \\
7 \\
1\end{array}$ & $\begin{array}{l}6 \\
5 \\
1\end{array}$ & $\begin{array}{l}5 \\
2\end{array}$ \\
\hline Anhui & Fuyang & $\begin{array}{l}\text { Cx. p. pallens } \\
\text { Ae. albopictus }\end{array}$ & $\begin{array}{l}\mathrm{F} \\
\mathrm{M} \\
\mathrm{F}\end{array}$ & $\begin{array}{r}54 \\
63 \\
2\end{array}$ & 1 & & 1 \\
\hline Hebei & Shijiazhuang & $\begin{array}{l}\text { Cx. p. pallens } \\
\text { Ae. albopictus } \\
\text { Ar. subalbatus }\end{array}$ & $\begin{array}{l}\mathrm{F} \\
\mathrm{M} \\
\mathrm{F} \\
\mathrm{M} \\
\mathrm{F}\end{array}$ & $\begin{array}{r}6 \\
2 \\
57 \\
61 \\
2\end{array}$ & & & \\
\hline Yunnan & Yiwei & Cx. p. pallens & $\begin{array}{l}\mathrm{F} \\
\mathrm{M}\end{array}$ & $\begin{array}{l}27 \\
94\end{array}$ & 4 & & 4 \\
\hline Beijing & Beijing & $\begin{array}{l}\text { Cx. p. pallens } \\
\text { An. sinensis }\end{array}$ & $\begin{array}{l}\mathrm{F} \\
\mathrm{M} \\
\mathrm{F}\end{array}$ & $\begin{array}{r}63 \\
54 \\
2\end{array}$ & $\begin{array}{r}8 \\
12\end{array}$ & 1 & $\begin{array}{r}8 \\
11\end{array}$ \\
\hline Guangdong & Zhanjiang & $\begin{array}{l}\text { Ae. albopictus } \\
\text { Cx. p. pallens } \\
\text { An. sinensis }\end{array}$ & $\begin{array}{l}\mathrm{F} \\
\mathrm{M} \\
\mathrm{F} \\
\mathrm{M} \\
\mathrm{F} \\
\mathrm{M}\end{array}$ & $\begin{array}{r}10 \\
3 \\
68 \\
3 \\
5 \\
28\end{array}$ & 1 & 1 & \\
\hline Henan & Anyang & $\begin{array}{l}\text { Cx. p. pallens } \\
\text { Ae. albopictus }\end{array}$ & $\begin{array}{l}\mathrm{F} \\
\mathrm{M} \\
\mathrm{F} \\
\mathrm{M}\end{array}$ & $\begin{array}{r}35 \\
50 \\
29 \\
4\end{array}$ & $\begin{array}{l}2 \\
1\end{array}$ & $\begin{array}{l}2 \\
1\end{array}$ & \\
\hline Liaoning & Jinzhou & $\begin{array}{l}\text { Ae. albopictus } \\
\text { An. sinensis } \\
\text { Cx. p. pallens } \\
\text { Ar. subalbatus }\end{array}$ & $\begin{array}{l}\mathrm{F} \\
\mathrm{M} \\
\mathrm{F} \\
\mathrm{F} \\
\mathrm{M} \\
\mathrm{F} \\
\mathrm{M}\end{array}$ & $\begin{array}{r}7 \\
4 \\
6 \\
3 \\
29 \\
34 \\
36\end{array}$ & & & \\
\hline Gansu & Jingyuan & Cx. p. pallens & $\begin{array}{l}\mathrm{F} \\
\mathrm{M}\end{array}$ & $\begin{array}{l}23 \\
59 \\
14\end{array}$ & 3 & 3 & \\
\hline Sichuan & Chengdu & $\begin{array}{l}\text { Ae. albopictus } \\
\text { Cx. p. pallens }\end{array}$ & $\begin{array}{l}\mathrm{F} \\
\mathrm{M} \\
\mathrm{F}\end{array}$ & $\begin{array}{r}57 \\
58 \\
4\end{array}$ & 2 & 2 & \\
\hline Shandong & Heze & $\begin{array}{l}\text { Ae. albopictus } \\
\text { Cx. p. pallens }\end{array}$ & $\begin{array}{l}\mathrm{F} \\
\mathrm{M} \\
\mathrm{F} \\
\mathrm{M}\end{array}$ & $\begin{array}{r}55 \\
58 \\
6 \\
2\end{array}$ & & & \\
\hline & Liaocheng & $\begin{array}{l}\text { Cx. p. pallens } \\
\text { Ae. albopictus } \\
\text { An. sinensis }\end{array}$ & $\begin{array}{l}\mathrm{F} \\
\mathrm{M} \\
\mathrm{F} \\
\mathrm{M} \\
\mathrm{F}\end{array}$ & $\begin{array}{r}50 \\
63 \\
4 \\
1 \\
3\end{array}$ & $\begin{array}{l}2 \\
3\end{array}$ & 2 & $\begin{array}{l}2 \\
1\end{array}$ \\
\hline Jilin & Changchun & $\begin{array}{l}\text { Cx. p. pallens } \\
\text { Ae. albopictus }\end{array}$ & $\begin{array}{l}\mathrm{F} \\
\mathrm{M} \\
\mathrm{F}\end{array}$ & $\begin{array}{r}115 \\
1 \\
5\end{array}$ & 2 & & 2 \\
\hline
\end{tabular}


TABle 1. (CONTINUED)

\begin{tabular}{|c|c|c|c|c|c|c|c|}
\hline Province & City & Species & Gender & No. & Total & Rickettsia felis & Rickettsia bellii \\
\hline Jiangxi & Xingan & $\begin{array}{l}\text { Ae. albopictus } \\
\text { Cx. p. pallens } \\
\text { An. sinensis }\end{array}$ & $\begin{array}{l}\mathrm{F} \\
\mathrm{M} \\
\mathrm{F} \\
\mathrm{M} \\
\mathrm{F} \\
\mathrm{M}\end{array}$ & $\begin{array}{r}23 \\
29 \\
13 \\
20 \\
7 \\
10\end{array}$ & & & \\
\hline Chongqing & Chongqing & $\begin{array}{l}\text { Ar. subalbatus } \\
\text { Ae. albopictus } \\
\text { Cx. p. pallens }\end{array}$ & $\begin{array}{l}\mathrm{F} \\
\mathrm{M} \\
\mathrm{F} \\
\mathrm{M}\end{array}$ & $\begin{array}{r}42 \\
65 \\
10 \\
3\end{array}$ & $\begin{array}{l}2 \\
3\end{array}$ & $\begin{array}{l}2 \\
3\end{array}$ & \\
\hline Hunan & Zhuzhou & $\begin{array}{l}\text { Ae. albopictus } \\
\text { Ar. subalbatus }\end{array}$ & $\begin{array}{l}\mathrm{F} \\
\mathrm{M} \\
\mathrm{F}\end{array}$ & $\begin{array}{r}115 \\
2 \\
2\end{array}$ & 2 & 2 & \\
\hline Hubei & Wuhan & $\begin{array}{l}\text { Ar. subalbatus } \\
\text { Cx. p. pallens } \\
\text { Ae. albopictus }\end{array}$ & $\begin{array}{l}\mathrm{F} \\
\mathrm{M} \\
\mathrm{F} \\
\mathrm{M} \\
\mathrm{F} \\
\mathrm{M}\end{array}$ & $\begin{array}{r}34 \\
20 \\
21 \\
21 \\
10 \\
3\end{array}$ & 3 & 1 & 2 \\
\hline Shaanxi & Changzhi & $\begin{array}{l}\text { Cx. p. pallens } \\
\text { Ae. albopictus }\end{array}$ & $\begin{array}{l}\mathrm{F} \\
\mathrm{M} \\
\mathrm{F}\end{array}$ & $\begin{array}{r}99 \\
9 \\
3\end{array}$ & 1 & 1 & \\
\hline Guangxi & Nanning & $\begin{array}{l}\text { Ae. albopictus } \\
\text { Cx. p. pallens }\end{array}$ & $\begin{array}{l}\mathrm{F} \\
\mathrm{M} \\
\mathrm{F} \\
\mathrm{M}\end{array}$ & $\begin{array}{r}90 \\
18 \\
10 \\
2\end{array}$ & $\begin{array}{l}3 \\
2\end{array}$ & $\begin{array}{l}3 \\
2\end{array}$ & \\
\hline Tianjin & Tianjin & $\begin{array}{l}\text { Cx. p. pallens } \\
\text { Ae. albopictus }\end{array}$ & $\begin{array}{l}\mathrm{F} \\
\mathrm{M} \\
\mathrm{F} \\
\mathrm{M}\end{array}$ & $\begin{array}{r}83 \\
6 \\
20 \\
7\end{array}$ & 6 & 5 & 1 \\
\hline Shanxi & Yanan & $\begin{array}{l}\text { Cx. p. pallens } \\
\text { Ae. albopictus }\end{array}$ & $\begin{array}{l}\mathrm{F} \\
\mathrm{M} \\
\mathrm{F}\end{array}$ & $\begin{array}{r}17 \\
97 \\
1\end{array}$ & $\begin{array}{l}1 \\
2\end{array}$ & 1 & 2 \\
\hline Fujian & Longyan & $\begin{array}{l}\text { Ar. subalbatus } \\
\text { Cx. p. pallens } \\
\text { Ae. albopictus } \\
\text { An. sinensis }\end{array}$ & $\begin{array}{l}\mathrm{F} \\
\mathrm{M} \\
\mathrm{F} \\
\mathrm{M} \\
\mathrm{F} \\
\mathrm{F}\end{array}$ & $\begin{array}{r}77 \\
38 \\
1 \\
1 \\
1 \\
2\end{array}$ & & & \\
\hline Guizhou & Liupanshui & $\begin{array}{l}\text { Cx. tritaeniorhynchus } \\
\text { Ar. subalbatus } \\
\text { An. sinensis }\end{array}$ & $\begin{array}{l}\mathrm{F} \\
\mathrm{M} \\
\mathrm{F} \\
\mathrm{M} \\
\mathrm{F}\end{array}$ & $\begin{array}{r}70 \\
10 \\
18 \\
9 \\
5\end{array}$ & 3 & 3 & \\
\hline Heilongjiang & Haerbin & $\begin{array}{l}\text { Cx. p. pallens } \\
\text { An. sinensis }\end{array}$ & $\begin{array}{l}\mathrm{F} \\
\mathrm{M} \\
\mathrm{F}\end{array}$ & $\begin{array}{r}45 \\
62 \\
3\end{array}$ & $\begin{array}{r}2 \\
25\end{array}$ & & $\begin{array}{r}2 \\
25\end{array}$ \\
\hline Jiangsu & Yangzhou & $\begin{array}{l}\text { Cx. p. pallens } \\
\text { Ae. albopictus }\end{array}$ & $\begin{array}{l}\mathrm{F} \\
\mathrm{M} \\
\mathrm{F} \\
\mathrm{M}\end{array}$ & $\begin{array}{l}51 \\
40 \\
27 \\
20\end{array}$ & $\begin{array}{l}2 \\
1\end{array}$ & $\begin{array}{l}1 \\
1\end{array}$ & 1 \\
\hline
\end{tabular}

Cx. p. pallens: Culex pipiens pallens; Cx. tritaeniorhynchus: Culex tritaeniorhynchus; An. sinensis: Anopheles sinensis; Ar. subalbatus: Aedes albopictus; Ar. subalbatus: Armigeres subalbatus.

2012a) and Ae. albopictus in Africa (Socolovschi et al. 2012b). Our study has confirmed the presence of $R$. felis in $C x$ pipiens pallens, An. Sinensis, and Ae. albopictus and has shown that the organism can also occur in Ar. subalbatus, a species found widely in China, Japan, and Southeast Asia
(Chaves et al. 2015). The females feed primarily on people and are the natural vectors of filarial worms (Muslim et al. 2013). Our findings that $R$. felis occurs widely in China in an increasing number of mosquito species that are known to feed on people should alert health workers to the possibility of 
FIG. 2. Molecular phyloettsia felis and Rickettsia bellii identified in this study. Distances and groupings of $R$. felis and $R$. bellii detected from the mosquitoes in this study ( $R$. felis MK061744 from China; $R$. bellii $\mathrm{MK}$ 697586 from China) (bold font) and reference Rickettsia sequences from NCBI ( $R$. felis KX446943 from Caratinga of Brazil; R. Bellii KU586331 from Beijing of China; Rickettsia sp. KY799071 from Gyeonggi of South Korea) were determined by applying the neighbor-joining method to a matrix of pairwise distances estimated using the MCL approach with MEGA version 6 software based on the gltA gene (443 bp). Scale bar indicates a genetic distance of 0.05-nt substitutions per position. MCL, maximum composite likelihood. genetic analysis of Rick-

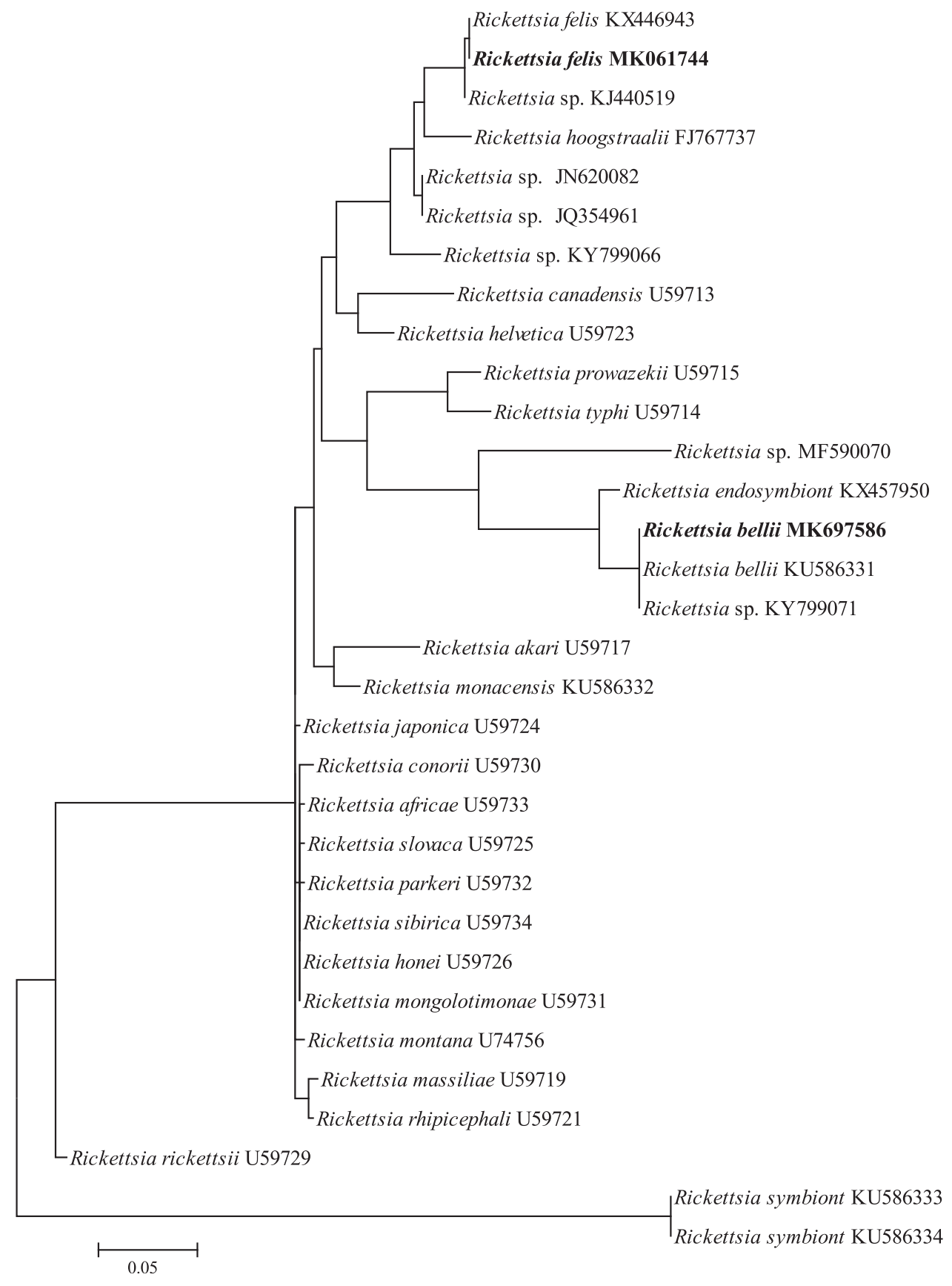

mosquito-transmitted infections in their patients. However, there are only limited data on the transmission of $R$. felis by mosquitoes (Dieme et al. 2015, Legendre and Macaluso 2017),

Previously, evidence has been provided for vertical transmission of $R$. felis in mosquitoes: the organism was recovered in the ovaries of experimentally infected An. gambiae (Dieme et al. 2015) as well as in a male An. arabiensis (Mediannikov et al. 2013). In each mosquito species found to be infected, similar numbers of males and females were positive for $R$. felis, overall 1.5\% (27/1809) and 1.8\% (22/ 1242), respectively. As male mosquitoes do not take blood meals (Nikbakhtzadeh et al. 2016), they most likely become infected by vertical transmission. Our data then provide further support for transovarial transmission of $R$. felis in mosquitoes (Dieme et al. 2015) and indicate this occurs across a wide range of mosquito species. In vivo infection studies are required to determine the roles of different mosquito species and hosts in the epidemiology of $R$. felis in Asia and its transmission to people.

Until recently, $R$. bellii has been considered to occur in a basal group of rickettsiae found in a wide range of hard and soft ticks in the Americas (Krawczak et al. 2018). Recent studies, however, have identified $R$. bellii or closely related organisms in ticks from Thailand (Sumrandee et al. 2016) and China (Song et al. 2018), An. sinensis in Zhejiang, China (Guo et al. 2016), and pools of Cx. pipiens from Korea (Maina et al. 2017). Our finding of $R$. bellii in mosquitoes from 10 provinces in China indicates that the organisms are probably widespread in mosquitoes in the region. As with $R$. felis, we found almost similar numbers of male and female C. pipiens pallens infected with $R$. bellii $(1.2 \%, 21 / 1809$; 
$4.0 \%, 48 / 1242$, respectively), and hence, this organism might also be transmitted transovarially. Further studies are indicated to more precisely determine the epidemiology of $R$. bellii in Asia and its significance in terms of animal and human health.

\section{Conclusions}

The present study revealed the presence of five common mosquito species, Culex pipiens pallens, Aedes albopictus, Armigeres subalbatus, Anopheles sinensis, and Culex tritaeniorhynchus, in and around domestic dwellings in 25 provinces of China. Both male and female mosquitoes from multiple provinces were found infected with $R$. felis or $R$. bellii indicating that the organisms are likely to be transmitted transovarially.

\section{Acknowledgments}

This project was supported by a grant from the National Natural Science Foundation of China (no: 31472225) and the Priority Academic Program Development of Jiangsu Higher Education Institutions, Yangzhou, Jiangsu, P. R. China, and by the Alabama Agricultural Experimental Station and the USDA National Institute of Food and Agriculture, Hatch project (ALA052-1-17026).

\section{Authors' Contribution Statement}

C.W. and J.Z. designed the research. J.Z. and G.L. collected the samples. J.Z., J.L., M.L., J.W., K.H., H.Q., J.Y., R.Z., Y.W., and Y.Z. performed the research. J.Z. and C.W. analyzed the data. J.Z., C.W., P.K., and H.W. prepared the article.

\section{Author Disclosure Statement}

No competing financial interests exist.

\section{References}

Blanton LS, Walker DH. Flea-Borne Rickettsioses and Rickettsiae. Am J Trop Med Hyg 2017; 96:53-56.

Cao Y, Fu S, Tian Z, Lu Z, et al. Distribution of mosquitoes and mosquito-borne arboviruses in Inner Mongolia, China. Vector Borne Zoonotic Dis 2011; 11:1577-1581.

Carvalho R, Vazquez D, Silveira P, Lencastre L. Rickettsiosis: A rare challenge in ICU. Intensive Care Med 2017; 43:1514 1515.

Chaves LF, Imanishi N, Hoshi T. Population dynamics of Armigeres subalbatus (Diptera: Culicidae) across a temperate altitudinal gradient. Bull Entomol Res 2015; 105: 589-597.

Dieme C, Bechah Y, Socolovschi C, Audoly G, et al. Transmission potential of Rickettsia felis infection by Anopheles gambiae mosquitoes. Proc Natl Acad Sci U S A 2015; 112: 8088-8093.

Fang LQ, Liu K, Li XL, Liang S, et al. Emerging tick-borne infections in mainland China: An increasing public health threat. Lancet Infect Dis 2015; 15:1467-1479.

Feng Y, Fu S, Zhang H, Li M, et al. Distribution of mosquitoes and mosquito-borne viruses along the China-Myanmar border in Yunnan Province. Jpn J Infect Dis 2012; 65:215221.
Folmer O, Black M, Hoeh W, Lutz R, et al. DNA primers for amplification of mitochondrial cytochrome $\mathrm{c}$ oxidase subunit I from diverse metazoan invertebrates. Mol Mar Biol Biotechnol 1994; 3:294-299.

Gillespie JJ, Beier MS, Rahman MS, Ammerman NC, et al. Plasmids and rickettsial evolution: Insight from Rickettsia felis. PLoS One 2007; 2:e266.

Gillespie JJ, Williams K, Shukla M, Snyder EE, et al. Rickettsia phylogenomics: Unwinding the intricacies of obligate intracellular life. PLoS One 2008; 3:e2018.

Guo WP, Tian JH, Lin XD, Ni XB, et al. Extensive genetic diversity of Rickettsiales bacteria in multiple mosquito species. Sci Rep 2016; 6:38770.

Guo XX, Li CX, Wang G, Zheng Z, et al. Host feeding patterns of mosquitoes in a rural malaria-endemic region in hainan island, china. J Am Mosq Control Assoc 2014; 30:309-311.

Guo Y, Song Z, Luo L, Wang Q, et al. Molecular evidence for new sympatric cryptic species of Aedes albopictus (Diptera: Culicidae) in China: A new threat from Aedes albopictus subgroup? Parasit Vectors 2018; 11:228.

Harbach, RE. Mosquito Taxonomic Inventory. 2013. http:// mosquito-taxonomic-inventory.info/ Accessed February 12, 2019.

Huang JX, Xia ZG, Zhou SS, Pu XJ, et al. Spatio-temporal analysis of malaria vectors in national malaria surveillance sites in China. Parasit Vectors 2015; 8:146.

Krawczak FS, Labruna MB, Hecht JA, Paddock CD, et al. Genotypic Characterization of Rickettsia bellii reveals distinct lineages in the United States and South America. Biomed Res Int 2018; 2018:8505483.

Legendre KP, Macaluso KR. Rickettsia felis: A Review of Transmission mechanisms of an emerging Pathogen. Trop Med Infect Dis 2017; 2:64.

Maina AN, Klein TA, Kim HC, Chong ST, et al. Molecular characterization of novel mosquito-borne Rickettsia spp. from mosquitoes collected at the Demilitarized Zone of the Republic of Korea. PLoS One 2017; 12:e0188327.

Mediannikov O, Socolovschi C, Edouard S, Fenollar F, et al. Common epidemiology of Rickettsia felis infection and malaria, Africa. Emerg Infect Dis 2013; 19:1775-1783.

Muslim A, Fong MY, Mahmud R, Lau YL, et al. Armigeres subalbatus incriminated as a vector of zoonotic Brugia pahangi filariasis in suburban Kuala Lumpur, Peninsular Malaysia. Parasit Vectors 2013; 6:219.

Nikbakhtzadeh MR, Buss GK, Leal WS. Toxic effect of blood feeding in male mosquitoes. Front Physiol 2016; 7:4.

Socolovschi C, Pages F, Ndiath MO, Ratmanov P, et al. Rickettsia species in African Anopheles mosquitoes. PLoS One 2012a; 7:e48254.

Socolovschi C, Pages F, Raoult D. Rickettsia felis in Aedes albopictus mosquitoes, Libreville, Gabon. Emerg Infect Dis 2012b; 18:1687-1689.

Song S, Chen C, Yang M, Zhao S, et al. Diversity of Rickettsia species in border regions of northwestern China. Parasit Vectors 2018; 11:634.

Sumrandee C, Baimai V, Trinachartvanit W, Ahantarig A. Molecular detection of Rickettsia, Anaplasma, Coxiella and Francisella bacteria in ticks collected from Artiodactyla in Thailand. Ticks Tick Borne Dis 2016; 7:678-689.

Wang G, Li C, Guo X, Xing D, et al. Identifying the main mosquito species in China based on DNA barcoding. PLoS One 2012; 7:e47051.

Wang J, Zhang H, Sun X, Fu S, et al. Distribution of mosquitoes and mosquito-borne arboviruses in Yunnan Province near the 
China-Myanmar-Laos border. Am J Trop Med Hyg 2011; 84: 738-746.

Wei L, Kelly P, Zhang J, Yang Y, et al. Use of a universal hydroxymethylbilane synthase (HMBS)-based PCR as an endogenous internal control and to enable typing of mammalian DNAs. Appl Microbiol Biotechnol 2014; 98:55795587.

Zhang J, John Kelly P, Lu G, Cruz-Martinez L, et al. Rickettsia in mosquitoes, Yangzhou, China. Emerg Microbes Infect 2016; 5:e108.

Zhang J, Lu G, Kelly P, Zhang Z, et al. First report of Rickettsia felis in China. BMC Infect Dis 2014; 14:682.
Zhang J, Wang J, Chen L, Yassin AK, et al. Housefly (Musca domestica) and Blow Fly (Protophormia terraenovae) as vectors of bacteria carrying colistin resistance genes. Appl Environ Microbiol 2018; 84:e1736-17.

Address correspondence to: Chengming Wang College of Veterinary Medicine Auburn University Auburn, AL 36849

E-mail: wangche@auburn.edu 